\title{
The Stability of the Solutions for a Porous Medium Equation with a Convection Term
}

\author{
Huashui Zhan (1) and Miao Ouyang (D) \\ School of Applied Mathematics, Xiamen University of Technology, Xiamen 361024, China \\ Correspondence should be addressed to Miao Ouyang; mouyang@xmut.edu.cn
}

Received 28 June 2017; Revised 17 November 2017; Accepted 22 November 2017; Published 10 January 2018

Academic Editor: Guang Zhang

Copyright (C) 2018 Huashui Zhan and Miao Ouyang. This is an open access article distributed under the Creative Commons Attribution License, which permits unrestricted use, distribution, and reproduction in any medium, provided the original work is properly cited.

\begin{abstract}
This paper studies the initial-boundary value problem of a porous medium equation with a convection term. If the equation is degenerate on the boundary, then only a partial boundary condition is needed generally. The existence of the weak solution is proved by the monotone convergent method. Moreover, according to the different boundary value conditions, the stability of the solutions is studied. In some special cases, the stability can be proved without any boundary value condition.
\end{abstract}

\section{Introduction}

Consider the motion of the ideal barotropic gas through a porous medium. Let $\rho$ be the gas density, $V$ the velocity, and $p$ the pressure. The motion is governed by the mass conservation law

$$
\rho_{t}+\operatorname{div}(\rho V)=0,
$$

the Darcy law

$$
V=-k(x) \nabla p,
$$

and the equation of stage $p=P(\rho)$. Here, $k(x)$ is a given matrix. We usually assume that $P(s)=\mu s^{\alpha}$ with $\mu, \alpha=$ const. The above laws then lead to a semilinear parabolic equation for the density $\rho$ :

$$
\rho_{t}=\frac{\mu \alpha}{1+\alpha} \operatorname{div}\left(k(x) \nabla \rho^{1+\alpha}\right) .
$$

If $k(x)=a(x) I$, where $a(x)$ is a function and $I$ is the unit matrix, then (3) becomes

$$
\rho_{t}=\frac{\mu \alpha}{1+\alpha} \operatorname{div}\left(a(x) \nabla \rho^{1+\alpha}\right)=\mu \alpha \operatorname{div}\left(a(x) \rho^{\alpha} \nabla \rho\right) .
$$

Also, (4) can be regarded as the generalization of the nonlinear heat equation

$$
u_{t}=\operatorname{div}(h(u, x) \nabla u),
$$

where the function $h(u, x)$ has the meaning of nonlinear thermal conductivity dependent on the temperature $u=$ $u(x, t)$. If $a(x) \equiv 1$ in (4) or $h(u, x) \equiv h(u)$ in (5), that is,

$$
u_{t}=\Delta u^{m},
$$

which is called the porous medium equation, there are well-known monographs or textbooks devoting to the wellposedness problem of (6); one can refer to [1-6] and the references therein. If $a(x) \geq 0$ in (4) or $h(u, x)$ depending on $x$ in (5), the situation may be different from that of (6). For example, if $\left.a(x)\right|_{x \in \partial \Omega}=0$, we consider the equation

$$
u_{t}=\nabla(a(x) \nabla u),
$$

and suppose that there are two classical solutions $u$ and $v$ of (7) with the initial values $u_{0}$ and $v_{0}$, respectively. Then it is easy to show that

$$
\int_{\Omega}|u(x, t)-v(x, t)|^{2} d x \leq \int_{\Omega}\left|u_{0}(x)-v_{0}(x)\right|^{2} d x,
$$

which implies that the classical solutions (if there are) of (7) are controlled by the initial value completely. In other words, the stability of the classical solutions of (7) is true without any boundary value condition. Yin and Wang [7] also showed that the non-Newtonian fluid equation with the type

$$
u_{t}=\operatorname{div}\left(d^{\alpha}(x)|\nabla u|^{p-2} \nabla u\right), \quad(x, t) \in \Omega \times(0, T)
$$


has similar properties, where $\Omega$ is a bounded domain in $R^{N}$ with appropriately smooth boundary, $d(x)=\operatorname{dist}(x, \partial \Omega)$, and $\alpha>0$ is a constant. Since the diffusion coefficient $d^{\alpha}(x)$ vanishes on the boundary, it seems that there is no heat flux across the boundary. However, Yin and Wang [7] showed that the fact might not coincide with what we image. In fact, the exponent $\alpha$, which characterizes the vanishing ratio of the diffusion coefficient near the boundary, does determine the behavior of the heat transfer near the boundary. They proved that, if $0<\alpha<p-1$, the solution of (9), $u \in H_{0}^{\gamma}$ for some constant $\gamma>1$, and the trace of $u$ on the boundary can be defined in the traditional way; then, in physics sense, there is no heat flux across the boundary actually, while, if $\alpha \geq$ $p-1$, the existence and uniqueness of solutions were proved without any boundary conditions, which means that whether there is heat flux across the boundary is uncertain. Later, Yin and Wang [8] had shown that only a partial boundary value condition matches up with the equation

$$
\begin{aligned}
\frac{\partial u}{\partial t} & -\operatorname{div}\left(a(x)|\nabla u|^{p-2} \nabla u\right)-f_{i}(x) D_{i} u+c(x, t) u \\
& =g(x, t), \quad(x, t) \in \Omega \times(0, T) .
\end{aligned}
$$

Inspired by Yin and Wang $[7,8]$, we will study the porous medium equation with a convection term,

$$
\begin{aligned}
& u_{t}=\operatorname{div}\left(d^{\alpha} \nabla u^{m}\right)+\sum_{i=1}^{N} \frac{\partial b_{i}\left(u^{m}\right)}{\partial x_{i}}, \\
& \quad(x, t) \in Q_{T}=\Omega \times(0, T),
\end{aligned}
$$

with the initial value

$$
u(x, 0)=u_{0}(x), \quad x \in \Omega,
$$

and with the partial boundary condition

$$
u(x, t)=0, \quad(x, t) \in \Sigma_{m} \times(0, T),
$$

where $\Sigma_{m}$ is defined as follows. When $0<\alpha<1, \Sigma_{m}=\partial \Omega$; when $\alpha \geq 1, \Sigma_{m}=\left\{x \in \partial \Omega: b_{i}^{\prime}(0) n_{i}(x)<0\right\}$ and $\left\{n_{i}\right\}$ is the inner normal vector of $\Omega$. The expression of $\Sigma_{m}$ is derived in [9], we do not repeat the details here.

We suppose that $b_{i}(s)$ is a $C^{1}$ function, and

$$
d^{\alpha / 2} \nabla u_{0}^{m} \in L^{2}(\Omega), \quad 0 \leq u_{0} \in L^{\infty}(\Omega) .
$$

Definition 1. A nonnegative function $u(x, t)$ is said to be the weak solution of (11) with the initial value (12), if

$$
d^{\alpha / 2}\left|\nabla u^{m}\right| \in L^{\infty}\left(0, T ; L^{2}(\Omega)\right), \quad u \in L^{\infty}\left(Q_{T}\right) ;
$$

for any function $\varphi \in C^{1}\left(Q_{T}\right),\left.\varphi\right|_{t=T}=0,\left.\varphi\right|_{\partial \Omega}=0$, there holds

$$
\begin{aligned}
& \iint_{Q_{T}}\left(-\frac{\partial \varphi}{\partial t} u+d^{\alpha} \nabla u^{m} \nabla \varphi\right) d x d t \\
& \quad+\iint_{Q_{T}} b_{i}\left(u^{m}\right) \varphi_{x_{i}}(x, t) d x d t \\
& =\int_{\Omega} u_{0} \varphi(x, 0) d x,
\end{aligned}
$$

and the initial condition is satisfied in the sense that

$$
\lim _{t \rightarrow 0} \int_{\Omega}\left|u(x, t)-u_{0}(x)\right| d x=0
$$

If $u(x, t)$ satisfies (13) in the sense of the trace in addition, then we say it is a weak solution of the initial-boundary value problem of (11).

First of all, we will study the well-posedness problem of (11).

Theorem 2. If $m>0,2>\alpha>0, u_{0}(x) \geq 0$ satisfy (15), then (11) with initial value (12) has a nonnegative solution. Moreover, if $0<\alpha<1$, then $\Sigma_{m}=\partial \Omega$; the solution is unique.

Then, we will study the stability of the solutions.

Theorem 3. If $b_{i}(u) \equiv 0$, i.e. equation (11) is not with the convection term, $u$ and $v$ are two solutions of equation (11) with the initial value $u_{0}(x), v_{0}(x)$ respectively, $\alpha>1$, then

$$
\int_{\Omega}|u(x, t)-v(x, t)| \leq c \int_{\Omega}\left|u_{0}(x)-v_{0}(x)\right| d x .
$$

Since $b_{i}(u) \equiv 0$ in Theorem 3 , there are some regrets more or less. For (11) itself, we can not prove the same conclusion for the time being. However, as compensation, we can consider a more complicate equation than (11),

$$
u_{t}=\operatorname{div}\left(d^{\alpha} \nabla u^{m}\right)+\sum_{i=1}^{N} \frac{\partial b_{i}\left(u^{m}, x, t\right)}{\partial x_{i}} .
$$

Theorem 4. Let $u$ and $v$ be two solutions of (19) with the initial values $u_{0}(x), v_{0}(x)$, respectively, if $1<\alpha<2$, and

$$
\left|b_{i}(\cdot, x, t)\right| \leq a(x),\left.\quad a(x)\right|_{x \in \partial \Omega}=0 ;
$$

$a(x)$ satisfies

$$
\int_{\Omega} a(x) d^{-\alpha} d x \leqslant c
$$

then the stability of the weak solutions is true in the sense of (18).

It is more or less strange that the case $\alpha=1$ is not included in Theorems 3 and 4.

At last, we will probe the stability of the weak solutions based on the partial boundary value condition.

Theorem 5. Let $u, v$ be two solutions of (11) with the initial values $u_{0}(x), v_{0}(x)$, respectively. If $2>\alpha \geq 1, m>0$,

$$
\begin{aligned}
& \int_{\Omega} d^{\alpha-1}\left|\nabla u^{m}\right|<\infty \\
& \int_{\Omega} d^{\alpha-1}\left|\nabla v^{m}\right|<\infty
\end{aligned}
$$


and the partial boundary condition (12) is satisfied in the sense of trace, then

$$
\begin{aligned}
& \int_{\Omega}|u(x, t)-v(x, t)| d x \\
& \quad \leq c \int_{\Omega}\left|u_{0}(x)-v_{0}(x)\right| d x+\int_{\Sigma_{m}^{\prime}}\left|u^{m}-v^{m}\right| d \Sigma,
\end{aligned}
$$

where $\Sigma_{m}^{\prime}=\partial \Omega \backslash \Sigma_{m}$.

Theorem 6. If $\Omega$ is a $C^{2}$ domain, $\alpha \geq 2$, and $m \geq 1$, then (11) with the initial value $u_{0}$ and the partial boundary condition (13) has a BV solution. Moreover, let $u, v$ be two solutions of (11) with the different initial values $u_{0}(x), v_{0}(x)$, respectively. Then

$$
\begin{aligned}
\int_{\Omega}|u(x, t)-v(x, t)| \leq & \int_{\Omega}\left|u_{0}(x)-v_{0}(x)\right| d x \\
& +\int_{\Sigma_{m}^{\prime}}|u-v| d \Sigma,
\end{aligned}
$$

where $\Sigma_{m}^{\prime}=\partial \Omega \backslash \Sigma_{m}, \Sigma_{m}=\left\{x \in \partial \Omega: b_{i}^{\prime}(0) n_{i}(x)<0\right\}$, and $\left\{n_{i}\right\}$ is the inner normal vector of $\Omega$.

If $b_{i} \equiv 0$, Theorem 6 has been included in Theorem 3, while, if $b_{i} \equiv 0$ is not true, then Theorem 6 has its independent sense. Such phenomena that the solution of a degenerate parabolic equation may be free from the limitation of the boundary condition also can be found in [7-14]. We will use some ideas in $[9,14]$. The uniqueness of the weak solutions when $\Sigma_{m}=\partial \Omega$ had been proved in [14]. Since [14] was written in Chinese, for the completeness of the paper, we still give its proof in what follows. In addition, how to obtain the stability (23) without condition (22) is a very interesting problem. Last but not least, roughly speaking, in this paper, we can show that if $o<\alpha<1$ or $\alpha \geq 2$, then the weak solution $u$ can be defined the trace on the boundary in the traditional sense; it is surprising that if $1 \leq \alpha<2$, whether $u$ can be defined the trace on the boundary is unknown for the time being.

\section{The Well-Posedness Problem}

We consider the following regularized problem:

$$
\begin{gathered}
u_{n t}=\operatorname{div}\left(\left(d+\frac{1}{n}\right)^{\alpha} \nabla u_{n}^{m}\right)+\sum_{i=1}^{N} \frac{\partial b_{i}\left(u_{n}^{m}\right)}{\partial x_{i}}, \\
\quad(x, t) \in Q_{T}, \\
u_{n}(x, t)=\frac{1}{n}, \quad(x, t) \in S_{T}=\partial \Omega \times(0, T), \\
u_{n}(x, 0)=u_{0 n}(x)=u_{0}(x)+\frac{1}{n}, \quad x \in \Omega .
\end{gathered}
$$

According to the standard parabolic equation theory, there is a weak solution

$$
\begin{array}{r}
u_{n} \in L^{\infty}\left(Q_{T}\right), \\
\nabla u_{n}^{m} \in L^{2}\left(Q_{T}\right),
\end{array}
$$

which satisfies

$$
\frac{1}{n} \leqslant u_{n}(x, t) \leq\left\|u_{0}\right\|_{L^{\infty}(\Omega)}+\frac{1}{n}, \quad(x, t) \in Q_{T},
$$

by the maximum principle.

Theorem 7. If $m>0,2>\alpha>0$, and $u_{0}(x) \geq 0$ satisfy (14), then (11) with initial value (12) has a nonnegative solution.

Proof. First we suppose that $u_{0} \in C_{0}^{\infty}(\Omega)$ and $0 \leq u_{0} \leq M$, and consider the following normalized problem

$$
u_{n t}=\operatorname{div}\left(a_{n}\left(u_{n}\right) \nabla u_{n}\right)+\sum_{i=1}^{N} \frac{\partial b_{i}\left(u_{n}^{m}\right)}{\partial x_{i}}
$$

$$
(x, t) \in Q_{T} \text {, }
$$

$$
\begin{aligned}
& u_{n}(x, t)=\frac{1}{n}, \quad(x, t) \in \partial \Omega \times(0, T), \\
& u_{n}(x, 0)=u_{0 n}(x), \quad x \in \Omega .
\end{aligned}
$$

Here, $a_{n}(u) \geqslant c(n)>0$, and

$$
a_{n}(u)=m\left(d+\frac{1}{n}\right)^{\alpha} u^{m-1}, \quad \text { if } u \in\left[\frac{1}{n}, M+\frac{1}{n}\right] .
$$

Thus, the solution of the problem $u_{n}$ is also a solution of problem (25). Moreover, by comparison theorem, we clearly have

$$
u_{n+1}(x, t) \leqslant u_{n}(x, t)
$$

which yields

$$
u(x, t)=\lim _{n \rightarrow \infty} u_{n}(x, t) .
$$

Now, we can prove that the limit function $u$ is a weak solution of (6) with the initial value (8).

Multiplying both sides of the first equation in (25) by $\phi=$ $u_{n}^{m}-(1 / n)^{m}$ and integrating it over $Q_{T}$, we have

$$
\begin{aligned}
& \int_{Q_{T}} u_{n t}\left(u_{n}^{m}-\left(\frac{1}{n}\right)^{m}\right) d x d t \\
& =\left.\int_{\Omega} u_{n}\left(u_{n}^{m}-\left(\frac{1}{n}\right)^{m}\right)\right|_{t=0} ^{t=T} d x \\
& \quad-\iint_{Q_{T}} u_{n} \frac{\partial}{\partial t}\left(u_{n}^{m}-\left(\frac{1}{n}\right)^{m}\right) d x d t \\
& =\int_{\Omega} u_{n}(x, T)\left(u_{n}^{m}(x, T)-\left(\frac{1}{n}\right)^{m}\right) d x \\
& \quad-\int_{\Omega} u_{0 n}(x)\left(u_{0 n}^{m}(x)-\left(\frac{1}{n}\right)^{m}\right) d x \\
& \quad-\iint_{Q_{T}} m u_{n}^{m} u_{n t} d x d t \\
& =\iint_{Q_{T}} \operatorname{div}\left[\left(d+\frac{1}{n}\right)^{\alpha} \nabla u_{n}^{m}\right]\left(u_{n}^{m}-\frac{1}{n^{m}}\right) d x d t \\
& +\sum_{i=1}^{N} \iint_{Q_{T}} \frac{\partial b_{i}\left(u_{n}^{m}\right)}{\partial x_{i}}\left(u_{n}^{m}-\left(\frac{1}{n}\right)^{m}\right) d x d t .
\end{aligned}
$$


By the fact

$$
\begin{aligned}
& \iint_{Q_{T}} \frac{\partial b_{i}\left(u_{n}^{m}\right)}{\partial x_{i}}\left(u_{n}^{m}-\left(\frac{1}{n}\right)^{m}\right) d x d t \\
& =-\iint_{Q_{T}} b_{i}\left(u_{n}^{m}\right) \frac{\partial}{\partial x_{i}}\left(u_{n}^{m}-\left(\frac{1}{n}\right)^{m}\right) d x d t \\
& =-\iint_{Q_{T}} \frac{\partial}{\partial x_{i}} \int_{(1 / n)^{m}}^{u_{n}^{m}} b_{i}(s) d s d x d t=0,
\end{aligned}
$$

then we have

$$
\begin{aligned}
& (m+1) \iint_{Q_{T}}\left(d+\frac{1}{n}\right)^{\alpha}\left|\nabla u_{n}^{m}\right|^{2} d x d t \\
& =\int_{\Omega}\left(u_{0 n}^{m}(x)-\frac{1}{n^{m}}\right) u_{0 n}(x) d x \\
& \quad-\int_{\Omega} u_{n}(x, T)\left(u_{n}^{m}(x, T)-\frac{1}{n^{m}}\right) d x \\
& \quad+\iint_{Q_{T}} \frac{\partial b_{i}\left(u_{n}^{m}\right)}{\partial x_{i}}\left(u_{n}^{m}-\left(\frac{1}{n}\right)^{m}\right) d x d t \\
& \leqslant \int_{\Omega}\left(u_{0}^{m}(x)+\frac{1}{n}\right)^{m+1} d x+\frac{1}{n}\left(M+\frac{1}{n}\right) \int_{\Omega} d x .
\end{aligned}
$$

Thus, we obtain

$$
\left|\left(d+\frac{1}{n}\right)^{\alpha / 2} \nabla u_{n}^{m}\right|_{L^{2}\left(Q_{T}\right)} \leq c .
$$

By choosing a subsequence, we can assume that

$$
\left(d+\frac{1}{n}\right)^{\alpha / 2} \nabla u_{n}^{m} \rightarrow \zeta
$$

weakly in $L^{2}$. We need to prove that

$$
\zeta=d^{\alpha / 2} \nabla u^{m}
$$

For any $\forall \psi \in C_{0}^{\infty}(\Omega)$, denoting that $d_{n} \triangleq d+1 / n$, we have

$$
\begin{aligned}
\iint_{Q_{T}} d_{n}^{\alpha / 2} \nabla u_{n}^{m} \cdot \psi d x d t \\
=\iint_{Q_{T}} \nabla\left(d_{n}^{\alpha / 2} u_{n}^{m}\right) \cdot \psi d x d t \\
\quad-\frac{\alpha}{2} \iint_{Q_{T}} d_{n}^{\alpha / 2-1} \nabla d \cdot u_{n}^{m} \psi d x d t \\
=-\iint_{Q_{T}} d_{n}^{\alpha / 2} u_{n}^{m} \cdot \nabla \psi d x d t \\
\quad-\frac{\alpha}{2} \iint_{Q_{T}} d_{n}^{\alpha / 2-1} \nabla d \cdot u_{n}^{m} \psi d x d t .
\end{aligned}
$$

Let $n \rightarrow \infty$. The left hand side is

$$
\lim _{n \rightarrow \infty} \iint_{Q_{T}} d_{n}^{\alpha / 2} \nabla u_{n}^{m} \cdot \psi d x d t=\iint_{Q_{T}} \zeta \psi d x d t .
$$

while on the right hand side, by

$$
\left|\nabla d \cdot u_{n}^{m}\right| \leq c|\nabla d| \cdot\left|u_{n}^{m}\right| \leq c\left(M+\frac{1}{n}\right)^{m} \leq c,
$$

and by the condition $0<\alpha<2$,

$$
\int_{\Omega} d_{n}^{\alpha / 2-1} d x \leq c,
$$

using the control convergent theorem,

$$
\begin{array}{r}
\frac{\alpha}{2} \lim _{n \rightarrow \infty} \iint_{Q_{T}} d_{n}^{\alpha / 2-1} \nabla d \cdot u_{n}^{m} \cdot \psi d x d t \\
=\frac{\alpha}{2} \iint_{Q_{T}} d^{\alpha / 2-1} \nabla d \cdot u^{m} \psi d x d t
\end{array}
$$

we have

$$
\begin{aligned}
& \lim _{n \rightarrow \infty}\left[-\iint_{Q_{T}} d_{n}^{\alpha / 2} u_{n}^{m} \cdot \nabla \psi d x d t\right. \\
& \left.-\frac{\alpha}{2} \iint_{Q_{T}} d_{n}^{\alpha / 2-1} \nabla d \cdot u_{n}^{m} \psi d x d t\right]=-\iint_{Q_{T}} d^{\alpha / 2} u^{m} \\
& \cdot \nabla \psi d x d t-\frac{\alpha}{2} \iint_{Q_{T}} d^{\alpha / 2-1} \nabla d \cdot u^{m} \psi d x d t \\
& =\iint_{Q_{T}} d^{\alpha / 2} \nabla u^{m} \cdot \psi d x d t .
\end{aligned}
$$

Thus we obtain (37).

At the same time, since $b_{i} \in C^{1}$, by (31), we have

$$
\lim _{n \rightarrow \infty} b_{i}\left(u_{n}^{m}\right)=b_{i}\left(u^{m}\right) \text {. }
$$

Thus, $u$ is a solution of (11) with the initial value (12).

If $u_{0}$ only satisfies (14), by considering the problem of (25) with the initial value $u_{0 \varepsilon}$ which is the mollified function of $u_{0}$, then we can get the conclusion by a process of limitation. Certainly, the solution $u(x, t)$ generally is not continuous at $t=0$, but satisfies (15) and (17). Theorem 7 is proved.

Lemma 8. Let $u_{0}$ satisfy (14). If $0<\alpha<1$ and $u$ is a solution of (11) with the initial value (12), then there exists a constant $\gamma>1$ such that

$$
\iint_{Q_{T}}\left|\nabla u^{m}\right|^{\gamma} d x d t \leq c .
$$

Proof. Since $\alpha<1$, there exists constant $\beta \in(\alpha, 1), \beta<(\alpha+$ $1) / 2$ such that $2-\alpha / \beta>1$. Therefore, there exists $\gamma \in(1,2-$ $\alpha / \beta)$ such that $\beta \gamma<1$. Therefore,

$$
\begin{aligned}
& \iint_{Q_{T}}\left|\nabla u^{m}\right|^{\gamma} d x d t \\
& =\iint_{\left\{(x, t) \in Q_{T} ; d^{\beta}\left|\nabla u^{m}\right| \leqslant 1\right\}}\left|\nabla u^{m}\right|^{\gamma} d x d t \\
& \quad+\iint_{\left\{(x, t) \in Q_{T} ; d^{\beta}\left|\nabla u^{m}\right|>1\right\}}\left|\nabla u^{m}\right|^{\gamma} d x d t \\
& \leqslant \iint_{Q_{T}} d^{-\beta \gamma} d x d t+\iint_{Q_{T}} d^{\alpha}\left|\nabla u^{m}\right|^{\alpha / \beta+\gamma} d x d t
\end{aligned}
$$




$$
\begin{aligned}
& \leqslant \iint_{Q_{T}} d^{-\beta \gamma} d x d t+\iint_{Q_{T}} d^{\alpha}\left(1+\left|\nabla u^{m}\right|^{2}\right) d x d t \\
& \leqslant c .
\end{aligned}
$$

Thus $u^{m}$ can be defined the trace on the boundary in the traditional way. By the definition of the trace, we also know that $u$ can be defined as the trace on the boundary in the traditional way. The lemma is proved.

Theorem 9. If $m>0,1>\alpha>0$ and $u_{0}(x) \geq 0$ satisfies (14), then $\Sigma_{m}=\partial \Omega$, and the solution of the initial-boundary value problem (11)-(13) is unique.

Proof. First of all, by Theorem 7 and Lemma 8, there is a nonnegative solution of the initial-boundary value problem (11)-(13). Then, we prove its uniqueness. Let $u, v$ be two solutions of equation (11) with

$$
\begin{aligned}
& u(x, 0)=v(x, 0), \\
& u(x, t)=v(x, t)=0, \quad(x, t) \in \partial \Omega \times(0, T) .
\end{aligned}
$$

For all $0 \leq \varphi \in C_{0}^{1}\left(Q_{T}\right)$,

$$
\begin{aligned}
\iint_{Q_{T}} \varphi & \frac{\partial(u-v)}{\partial t} d x d t \\
= & -\iint_{Q_{T}} d^{\alpha}\left(\nabla u^{m}-\nabla v^{m}\right) \cdot \nabla \varphi d x d t \\
& \quad-\sum_{i=1}^{N} \iint_{Q_{T}}\left[b_{i}\left(u^{m}\right)-b_{i}\left(v^{m}\right)\right] \varphi_{x_{i}} d x d t .
\end{aligned}
$$

For any given positive integer $n$, let $g_{n}(s)=\int_{0}^{s} h_{n}(\tau) d \tau$, $h_{n}(s)=2 n(1-n|s|)_{+}$. Then $h_{n}(s) \in C(\mathbb{R})$, and we have

$$
\begin{aligned}
h_{n}(s) & \geq 0, \\
\left|s h_{n}(s)\right| & \leq 1, \\
\left|g_{n}(s)\right| & \leq 1, \\
\lim _{n \rightarrow \infty} g_{n}(s) & =\operatorname{sgn} s, \\
\lim _{n \rightarrow \infty} s g_{n}^{\prime}(s) & =0 .
\end{aligned}
$$

Since $0<\alpha<1$, by Lemma 8 , we can define the traces of $u, v$ on the boundary. By a process of limit, we can choose $g_{n}\left(u^{m}-v^{m}\right)$ as the test function; then

$$
\begin{aligned}
& \int_{\Omega} g_{n}\left(u^{m}-v^{m}\right) \frac{\partial(u-v)}{\partial t} d x+\int_{\Omega} d^{\alpha}\left(\nabla u^{m}-\nabla v^{m}\right) \\
& \cdot \nabla\left(u^{m}-v^{m}\right) g_{n}^{\prime}\left(u^{m}-v^{m}\right) d x \\
& +\sum_{i=1}^{N} \iint_{Q_{T}}\left[b_{i}\left(u^{m}\right)-b_{i}\left(v^{m}\right)\right]\left(u^{m}-v^{m}\right)_{x_{i}} \\
& \cdot g_{n}^{\prime}\left(u^{m}-v^{m}\right) d x d t=0 .
\end{aligned}
$$

Moreover, we can prove that

$$
\begin{gathered}
\lim _{n \rightarrow \infty} \int_{\Omega}\left(b_{i}\left(u^{m}\right)-b_{i}\left(v^{m}\right)\right) g_{n}^{\prime}\left(u^{m}-v^{m}\right) \\
\cdot\left(u^{m}-v^{m}\right)_{x_{i}} d x=0 .
\end{gathered}
$$

In detail, the limitation (51) is established by the following calculations.

$$
\begin{aligned}
& \left|\int_{\Omega}\left(b_{i}\left(u^{m}\right)-b_{i}\left(v^{m}\right)\right) g_{n}^{\prime}\left(u^{m}-v^{m}\right)\left(u^{m}-v^{m}\right)_{x_{i}} d x\right| \\
& \quad=\mid \int_{\left\{x \in \Omega:\left|u^{m}-v^{m}\right|<1 / n\right\}}\left[b_{i}\left(u^{m}\right)-b_{i}\left(v^{m}\right)\right] g_{n}^{\prime}\left(u^{m}-v^{m}\right) \\
& \cdot\left(u^{m}-v^{m}\right)_{x_{i}} d x \mid \\
& \quad \leqslant c \int_{\left\{x \in \Omega:\left|u^{m}-v^{m}\right|<1 / n\right\}}\left|\frac{b_{i}\left(u^{m}\right)-b_{i}\left(v^{m}\right)}{u^{m}-v^{m}}\right|\left|\left(u^{m}-v^{m}\right)_{x_{i}}\right| d x \\
& \quad=c \int_{\left\{x \in \Omega:\left|u^{m}-v^{m}\right|<1 / n\right\}}\left|d^{-\alpha / 2} \frac{b_{i}\left(u^{m}\right)-b_{i}\left(v^{m}\right)}{u^{m}-v^{m}}\right| \\
& \quad \cdot\left|d^{\alpha / 2}\left(u^{m}-v^{m}\right)_{x_{i}}\right| d x \\
& \quad \leqslant c\left[\int_{\{x \in \Omega:|u-v|<1 / n\}}\left(\left|d^{-\alpha / 2} \frac{b_{i}\left(u^{m}\right)-b_{i}\left(v^{m}\right)}{u^{m}-v^{m}}\right|\right)^{2} d x\right]^{1 / 2} \\
& \quad \cdot\left[\int_{\left\{x \in \Omega:\left|u^{m}-v^{m}\right|<1 / n\right\}} d^{\alpha}\left|\nabla\left(u^{m}-v^{m}\right)\right|^{2} d x\right]^{1 / 2} \cdot
\end{aligned}
$$

Since $0<\alpha<1$,

$$
\begin{aligned}
& \int_{\left\{x \in \Omega:\left|u^{m}-v^{m}\right|<1 / n\right\}}\left(\left|d^{-\alpha / 2} \frac{b_{i}\left(u^{m}\right)-b_{i}\left(v^{m}\right)}{u^{m}-v^{m}}\right|\right)^{2} d x \\
& \leqslant c \int_{\left\{x \in \Omega:\left|u^{m}-v^{m}\right|<1 / n\right\}} d^{-\alpha}(x) d x \leqslant c \int_{\Omega} d^{-\alpha} d x \leqslant c .
\end{aligned}
$$

In (52), let $n \rightarrow \infty$. If $\left\{x \in \Omega:\left|u^{m}-v^{m}\right|=0\right\}$ is a set with 0 measure, by $0<\alpha<1$, we have

$$
\begin{aligned}
& \lim _{n \rightarrow \infty} \int_{\left\{x \in \Omega:\left|u^{m}-v^{m}\right|<1 / n\right\}} d^{-\alpha}(x) d x \\
& =\int_{\left\{x \in \Omega:\left|u^{m}-v^{m}\right|=0\right\}} d^{-\alpha}(x) d x=0 .
\end{aligned}
$$

If the set $\left\{x \in \Omega:\left|u^{m}-v^{m}\right|=0\right\}$ has a positive measure, then,

$$
\begin{aligned}
& \lim _{n \rightarrow \infty} \int_{\left\{x \in \Omega:\left|u^{m}-v^{m}\right|<1 / n\right\}} d^{\alpha}\left|\nabla\left(u^{m}-v^{m}\right)\right|^{2} d x \\
& =\int_{\left\{x \in \Omega:\left|u^{m}-v^{m}\right|=0\right\}} d^{\alpha}\left|\nabla\left(u^{m}-v^{m}\right)\right|^{2} d x=0 .
\end{aligned}
$$

Therefore, in both cases, the right hand side of inequality (52) goes to 0 as $n \rightarrow \infty$. 
Clearly,

$$
\begin{aligned}
& \lim _{n \rightarrow \infty} \int_{\Omega} g_{n}\left(u^{m}-v^{m}\right) \frac{\partial(u-v)}{\partial t} d x \\
& \quad=\int_{\Omega} \operatorname{sgn}\left(u^{m}-v^{m}\right) \frac{\partial(u-v)}{\partial t} d x \\
& \quad=\int_{\Omega} \operatorname{sgn}(u-v) \frac{\partial(u-v)}{\partial t}=\frac{d}{d t}\|u-v\|_{1} .
\end{aligned}
$$

Now, let $n \rightarrow \infty$ in (50). Then

$$
\begin{aligned}
& \int_{\Omega}|u(x, t)-v(x, t)| d x \leqslant \int_{\Omega}\left|u_{0}(x)-v_{0}(x)\right| d x \\
& \quad=0 .
\end{aligned}
$$

We have the conclusion.

By Theorems 7 and 9, we clearly have the following.

Corollary 10. Theorem 2 is true.

\section{The Stability without the Boundary Value Condition}

Consider a simpler equation than (11).

$$
u_{t}=\operatorname{div}\left(d^{\alpha} \nabla u^{m}\right), \quad(x, t) \in Q_{T}=\Omega \times(0, T),
$$

with the initial value (12), but without any boundary value condition. For a small positive constant $\lambda>0$, let

$$
\Omega_{\lambda}=\{x \in \Omega: d(x)=\operatorname{dist}(x, \partial \Omega)>\lambda\},
$$

and let

$$
\phi(x)= \begin{cases}1, & \text { if } x \in \Omega_{2 \lambda}, \\ \frac{1}{\lambda}(d(x)-\lambda), & x \in \Omega_{\lambda} \backslash \Omega_{2 \lambda} \\ 0, & \text { if } x \in \Omega \backslash \Omega_{\lambda} .\end{cases}
$$

Proof of Theorem 3. Suppose $u_{0}, v_{0}$ only satisfy (7), $\alpha>1$. Let $u, v$ be two solutions of (58) with the initial-boundary values $u_{0}, v_{0}$, respectively. For all $0 \leq \varphi \in C_{0}^{1}\left(Q_{T}\right)$,

$$
\begin{aligned}
& \iint_{Q_{T}} \varphi \frac{\partial(u-v)}{\partial t} d x d t \\
& \quad=-\iint_{Q_{T}} d^{\alpha}\left(\nabla u^{m}-\nabla v^{m}\right) \cdot \nabla \varphi d x d t .
\end{aligned}
$$

By a process of limit, we can choose $\phi g_{n}\left(u^{m}-v^{m}\right)$ as the test function; then

$$
\begin{aligned}
& \int_{\Omega} \phi(x) g_{n}\left(u^{m}-v^{m}\right) \frac{\partial(u-v)}{\partial t} d x \\
& \quad+\int_{\Omega} d^{\alpha}\left(\nabla u^{m}-\nabla v^{m}\right) \\
& \cdot \phi \nabla\left(u^{m}-v^{m}\right) g_{n}^{\prime}\left(u^{m}-v^{m}\right) d x \\
& \quad+\int_{\Omega} d^{\alpha}\left(\nabla u^{m}-\nabla v^{m}\right) \cdot \nabla \phi g_{n}\left(u^{m}-v^{m}\right) d x=0 .
\end{aligned}
$$

Clearly, we have

$$
\begin{aligned}
& \int_{\Omega} d^{\alpha}\left(\nabla u^{m}-\nabla v^{m}\right) \cdot \phi \nabla\left(u^{m}-v^{m}\right) g_{n}^{\prime}\left(u^{m}-v^{m}\right) d x \\
& \quad \geq 0 \\
& \lim _{n \rightarrow \infty} \lim _{\lambda \rightarrow 0} \int_{\Omega} \phi(x) g_{n}\left(u^{m}-v^{m}\right) \frac{\partial(u-v)}{\partial t} d x \\
& \quad=\lim _{n \rightarrow \infty} \int_{\Omega} g_{n}\left(u^{m}-v^{m}\right) \frac{\partial(u-v)}{\partial t} d x \\
& \quad=\int_{\Omega} \operatorname{sgn}\left(u^{m}-v^{m}\right) \frac{\partial(u-v)}{\partial t} d x \\
& \quad=\int_{\Omega} \operatorname{sgn}(u-v) \frac{\partial(u-v)}{\partial t}=\frac{d}{d t}\|u-v\|_{1} .
\end{aligned}
$$

As for the term

$$
\int_{\Omega} d^{\alpha}\left(\nabla u^{m}-\nabla v^{m}\right) \cdot \nabla \phi g_{n}\left(u^{m}-v^{m}\right) d x,
$$

we have

$$
\begin{gathered}
\lim _{\lambda \rightarrow 0}\left|\int_{\Omega} d^{\alpha}\left(\nabla u^{m}-\nabla v^{m}\right) \cdot \nabla \phi g_{n}\left(u^{m}-v^{m}\right) d x\right| \\
\leq\left(\int_{\Omega} d^{\alpha}\left(\left|\nabla u^{m}\right|^{2}+\left|\nabla v^{m}\right|^{2}\right) d x\right)^{1 / 2} \\
\cdot\left(\int_{\Omega_{\lambda} \backslash \Omega_{2 \lambda}} d^{\alpha}|\nabla \phi|^{2} d x\right)^{1 / 2}=0 .
\end{gathered}
$$

The last equality of (65) is due to that since $\alpha>1$, we have

$$
\begin{aligned}
& \lim _{\lambda \rightarrow 0} \int_{\Omega_{\lambda} \backslash \Omega_{2 \lambda}} d^{\alpha}|\nabla \phi|^{2} d x=4 \lim _{\lambda \rightarrow 0} \int_{\Omega_{\lambda} \backslash \Omega_{2 \lambda}} \lambda^{\alpha} \frac{|\nabla d|^{2}}{\lambda^{2}} d x \\
& \quad \leq c \lim _{\lambda \rightarrow 0} \lambda^{\alpha-1}=0 .
\end{aligned}
$$

Now, after letting $\lambda \rightarrow 0$, let $n \rightarrow \infty$ in (62). Then

$$
\int_{\Omega}|u(x, t)-v(x, t)| d x \leqslant \int_{\Omega}\left|u_{0}(x)-v_{0}(x)\right| d x .
$$

Theorem 3 is proved.

Consider a more complicated equation than (11).

$$
\begin{aligned}
& u_{t}=\operatorname{div}\left(d^{\alpha} \nabla u^{m}\right)+\sum_{i=1}^{N} \frac{\partial b_{i}\left(u^{m}, x, t\right)}{\partial x_{i}}, \\
&(x, t) \in Q_{T}=\Omega \times(0, T),
\end{aligned}
$$

with the initial value (12), but without any boundary value condition. 
Proof of Theorem 4. Suppose $u_{0}, v_{0}$ only satisfy (7), $1<\alpha<$ 2 . Let $u, v$ be two solutions of equation (11) with the initialboundary values $u_{0}, v_{0}$, respectively. For all $0 \leq \varphi \in C_{0}^{1}\left(Q_{T}\right)$,

$$
\begin{aligned}
\iint_{Q_{T}} \varphi & \frac{\partial(u-v)}{\partial t} d x d t \\
= & -\iint_{Q_{T}} d^{\alpha}\left(\nabla u^{m}-\nabla v^{m}\right) \cdot \nabla \varphi d x d t \\
& -\sum_{i=1}^{N} \iint_{Q_{T}}\left[b_{i}\left(u^{m}, x, t\right)-b_{i}\left(v^{m}, x, t\right)\right] \varphi_{x_{i}} d x d t .
\end{aligned}
$$

By a process of limit, we can choose $g_{n}\left(\phi\left(u^{m}-v^{m}\right)\right)$ as the test function; then

$$
\begin{aligned}
& \int_{\Omega} g_{n}\left(\phi\left(u^{m}-v^{m}\right)\right) \frac{\partial(u-v)}{\partial t} d x+\int_{\Omega} d^{\alpha}\left(\nabla u^{m}\right. \\
& \left.-\nabla v^{m}\right) \cdot \phi \nabla\left(u^{m}-v^{m}\right) g_{n}^{\prime}\left(\phi\left(u^{m}-v^{m}\right)\right) d x \\
& +\int_{\Omega} d^{\alpha}\left(\nabla u^{m}-\nabla v^{m}\right) \cdot \nabla \phi\left(u^{m}-v^{m}\right) g_{n}^{\prime}\left(u^{m}\right. \\
& \left.-v^{m}\right) d x \\
& +\sum_{i=1}^{N} \iint_{Q_{T}}\left[b_{i}\left(u^{m}, x, t\right)-b_{i}\left(v^{m}, x, t\right)\right] \\
& \cdot\left[\phi_{x_{i}}\left(u^{m}-v^{m}\right)+\phi\left(u^{m}-v^{m}\right)_{x_{i}}\right] \\
& \cdot g_{n}^{\prime}\left(\phi\left(u^{m}-v^{m}\right)\right) d x d t=0 .
\end{aligned}
$$

Let us analyze every term in the left hand side of (70). For the first term, we clearly have

$$
\begin{gathered}
\lim _{n \rightarrow \infty} \lim _{\lambda \rightarrow 0} \int_{\Omega} g_{n}\left(\phi\left(u^{m}-v^{m}\right)\right) \frac{\partial(u-v)}{\partial t} d x \\
=\lim _{n \rightarrow \infty} \int_{\Omega} g_{n}\left(u^{m}-v^{m}\right) \frac{\partial(u-v)}{\partial t} d x \\
=\int_{\Omega} \operatorname{sgn}\left(u^{m}-v^{m}\right) \frac{\partial(u-v)}{\partial t} d x \\
=\int_{\Omega} \operatorname{sgn}(u-v) \frac{\partial(u-v)}{\partial t}=\frac{d}{d t}\|u-v\|_{1} .
\end{gathered}
$$

For the second term, we have

$$
\begin{aligned}
& \int_{\Omega} d^{\alpha}\left(\nabla u^{m}-\nabla v^{m}\right) \\
& \cdot \phi \nabla\left(u^{m}-v^{m}\right) g_{n}^{\prime}\left(\phi\left(u^{m}-v^{m}\right)\right) d x \geq 0 .
\end{aligned}
$$

For the third term, since

$$
\begin{aligned}
& \lim _{\lambda \rightarrow 0} \int_{\Omega_{\lambda} \backslash \Omega_{2 \lambda}}\left|d^{\alpha / 2-1}\left(u^{m}-v^{m}\right) g_{n}^{\prime}\left(\phi\left(u^{m}-v^{m}\right)\right)\right|^{2} d x \\
& \quad \leq \lim _{\lambda \rightarrow 0} \int_{\Omega} d^{\alpha-2}\left|\left(u^{m}-v^{m}\right) g_{n}^{\prime}\left(\phi\left(u^{m}-v^{m}\right)\right)\right|^{2} d x \\
& \quad \leq \int_{\Omega} d^{\alpha-2} d x \leq c,
\end{aligned}
$$

by $0>\alpha-2>-1$, we have

$$
\begin{aligned}
& \lim _{\lambda \rightarrow 0} \int_{\Omega} d^{\alpha}\left(\nabla u^{m}-\nabla v^{m}\right) \cdot \nabla \phi\left(u^{m}-v^{m}\right) \\
& \cdot g_{n}^{\prime}\left(\phi\left(u^{m}-v^{m}\right)\right) d x \\
& \quad=\lim _{\lambda \rightarrow 0} \int_{\Omega_{\lambda} \backslash \Omega_{2 \lambda}} \frac{c}{\lambda} d^{\alpha}\left|\nabla u^{m}-\nabla v^{m}\right| \mid\left(u^{m}-v^{m}\right) \\
& \cdot g_{n}^{\prime}\left(\phi\left(u^{m}-v^{m}\right)\right) \mid d x \leq c \\
& \cdot \lim _{\lambda \rightarrow 0} \int_{\Omega_{\lambda} \backslash \Omega_{2 \lambda}} d^{\alpha-1}\left|\nabla u^{m}-\nabla v^{m}\right| \mid\left(u^{m}-v^{m}\right) \\
& \cdot g_{n}^{\prime}\left(\phi\left(u^{m}-v^{m}\right)\right) \mid d x \leq c \\
& \cdot \lim _{\lambda \rightarrow 0}\left(\int_{\Omega_{\lambda} \backslash \Omega_{2 \lambda}}\left[d^{\alpha / 2}\left|\nabla u^{m}-\nabla v^{m}\right|\right]^{2} d x\right)^{1 / 2} \\
& \cdot\left(\int_{\Omega_{\lambda} \backslash \Omega_{2 \lambda}} \mid d^{\alpha / 2-1}\left(u^{m}-v^{m}\right)\right. \\
& \left.\left.\cdot g_{n}^{\prime}\left(\phi\left(u^{m}-v^{m}\right)\right)\right|^{2} d x\right)^{1 / 2}=0,
\end{aligned}
$$

by $\lim _{n \rightarrow \infty} g_{n}^{\prime}(s) s=0$.

Now, we deal with the terms related to the convection function $b_{i}$ in (70). In the first place, by (20),

$$
\left|b_{i}\left(u^{m}, x, t\right)-b_{i}\left(v^{m}, x, t\right)\right| \leq 2 a(x) ;
$$

according to the definition of the trace, we have

$$
\begin{aligned}
& \lim _{\lambda \rightarrow 0} \mid \int_{\Omega}\left(b_{i}\left(u^{m}, x, t\right)-b_{i}\left(v^{m}, x, t\right)\right) g_{n}^{\prime}\left(\phi\left(u^{m}-v^{m}\right)\right) \\
& \cdot\left(u^{m}-v^{m}\right) \phi_{x_{i}}(x) d x \mid \leq \lim _{\lambda \rightarrow 0} \frac{c}{\lambda} \int_{\Omega_{\lambda} \backslash \Omega_{2 \lambda}} a(x) \\
& \cdot\left|u^{m}-v^{m}\right| g_{n}^{\prime}\left(\phi\left(u^{m}-v^{m}\right)\right) d x \leq c \int_{\partial \Omega} a(x) d \Sigma \\
& \quad=0 .
\end{aligned}
$$

Moreover, we can prove that

$$
\begin{aligned}
\lim _{n \rightarrow \infty} \lim _{\lambda \rightarrow 0} \int_{\Omega}\left(b_{i}\left(u^{m}, x, t\right)-b_{i}\left(v^{m}, x, t\right)\right) \\
\quad \cdot g_{n}^{\prime}\left(\phi\left(u^{m}-v^{m}\right)\right)\left(u^{m}-v^{m}\right)_{x_{i}} \phi(x) d x=0 .
\end{aligned}
$$

In detail, the limitation (77) is established by the following calculations. 


$$
\begin{aligned}
& \lim _{\lambda \rightarrow 0}\left|\int_{\Omega}\left(b_{i}\left(u^{m}, x, t\right)-b_{i}\left(v^{m}, x, t\right)\right) g_{n}^{\prime}\left(\phi\left(u^{m}-v^{m}\right)\right)\left(u^{m}-v^{m}\right)_{x_{i}} \phi(x) d x\right| \\
& \quad=\left|\int_{\Omega}\left(b_{i}\left(u^{m}, x, t\right)-b_{i}\left(v^{m}, x, t\right)\right) g_{n}^{\prime}\left(u^{m}-v^{m}\right)\left(u^{m}-v^{m}\right)_{x_{i}} d x\right| \\
& \quad=\left|\int_{\left\{x \in \Omega:\left|u^{m}-v^{m}\right|<1 / n\right\}}\left[b_{i}\left(u^{m}, x, t\right)-b_{i}\left(v^{m}, x, t\right)\right] g_{n}^{\prime}\left(u^{m}-v^{m}\right)\left(u^{m}-v^{m}\right)_{x_{i}} d x\right| \\
& \quad \leqslant c \int_{\left\{x \in \Omega:\left|u^{m}-v^{m}\right|<1 / n\right\}}\left|\frac{b_{i}\left(u^{m}, x, t\right)-b_{i}\left(v^{m}, x, t\right)}{u^{m}-v^{m}}\right|\left|\left(u^{m}-v^{m}\right)_{x_{i}}\right| d x \\
& \quad=c \int_{\left\{x \in \Omega:\left|u^{m}-v^{m}\right|<1 / n\right\}}\left|d^{-\alpha / 2} \frac{b_{i}\left(u^{m}, x, t\right)-b_{i}\left(v^{m}, x, t\right)}{u^{m}-v^{m}}\right|\left|d^{\alpha / 2}\left(u^{m}-v^{m}\right)_{x_{i}}\right| d x \\
& \quad \leqslant c\left[\int_{\{x \in \Omega:|u-v|<1 / n\}}\left(\left|d^{-\alpha / 2} \frac{b_{i}\left(u^{m}, x, t\right)-b_{i}\left(v^{m}, x, t\right)}{u^{m}-v^{m}}\right|\right)^{2} d x\right]^{1 / 2} \cdot\left[\int_{\left\{x \in \Omega:\left|u^{m}-v^{m}\right|<1 / n\right\}}\left|d^{\alpha / 2} \nabla\left(u^{m}-v^{m}\right)\right|^{2} d x\right]^{1 / 2} .
\end{aligned}
$$

By (21),

$$
\begin{aligned}
& \int_{\left\{x \in \Omega:\left|u^{m}-v^{m}\right|<1 / n\right\}}\left(\mid d^{-\alpha / 2}\right. \\
& \left.. \frac{b_{i}\left(u^{m}, x, t\right)-b_{i}\left(v^{m}, x, t\right)}{u^{m}-v^{m}} \mid\right)^{2} d x \\
& \leqslant c \int_{\Omega} a(x) d^{-\alpha} d x \leqslant c .
\end{aligned}
$$

Let $n \rightarrow \infty$ in (78). If $\left\{x \in \Omega:\left|u^{m}-v^{m}\right|=0\right\}$ is a set with 0 measure, then

$$
\begin{aligned}
\lim _{n \rightarrow \infty} & \int_{\left\{x \in \Omega:\left|u^{m}-v^{m}\right|<1 / n\right\}} a(x) d^{-\alpha} d x \\
= & \int_{\left\{x \in \Omega:\left|u^{m}-v^{m}\right|=0\right\}} a(x) d^{-\alpha} d x=0 .
\end{aligned}
$$

If the set $\left\{x \in \Omega:\left|u^{m}-v^{m}\right|=0\right\}$ has a positive measure, then,

$$
\begin{aligned}
& \lim _{n \rightarrow \infty} \int_{\left\{x \in \Omega:\left|u^{m}-v^{m}\right|<1 / n\right\}} d^{\alpha}\left|\nabla\left(u^{m}-v^{m}\right)\right|^{2} d x \\
& =\int_{\left\{x \in \Omega:\left|u^{m}-v^{m}\right|=0\right\}} d^{\alpha}\left|\nabla\left(u^{m}-v^{m}\right)\right|^{2} d x=0 .
\end{aligned}
$$

Therefore, in both cases, the right hand side of inequality (74) goes to 0 as $n \rightarrow \infty$.

At last, Now, after letting $\lambda \rightarrow 0$, let $n \rightarrow \infty$ in (71). By (72), (73), (74), (76), (77), (78), (79), (80), and (81), then

$$
\int_{\Omega}|u(x, t)-v(x, t)| d x \leqslant \int_{\Omega}\left|u_{0}(x)-v_{0}(x)\right| d x .
$$

Theorem 4 is proved.

\section{The Stability Based on the Partial Boundary Value Condition}

In this section, we will prove Theorem 5; the proof is similar as that of Theorem 4
Proof of Theorem 5. Suppose $u_{0}$, $v_{0}$ only satisfy (7), $1 \leq \alpha<$ 2. Let $u, v$ be two solutions of (11) with the initial-boundary values $u_{0}, v_{0}$, respectively, and with the same homogeneous partial boundary value condition

$$
u(x, t)=v(x, t)=0, \quad(x, t) \in \Sigma_{m} \times(0, T),
$$

For all $0 \leq \varphi \in C_{0}^{1}\left(Q_{T}\right)$

$$
\begin{aligned}
& \iint_{Q_{T}} \varphi \frac{\partial(u-v)}{\partial t} d x d t \\
& =-\iint_{Q_{T}} d^{\alpha}\left(\nabla u^{m}-\nabla v^{m}\right) \cdot \nabla \varphi d x d t \\
& \quad-\sum_{i=1}^{N} \iint_{Q_{T}}\left[b_{i}\left(u^{m}\right)-b_{i}\left(v^{m}\right)\right] \varphi_{x_{i}} d x d t .
\end{aligned}
$$

By a process of limit, we can choose $g_{n}\left(\phi\left(u^{m}-v^{m}\right)\right)$ as the test function as in Theorem 4; then

$$
\begin{aligned}
& \int_{\Omega} g_{n}\left(\phi\left(u^{m}-v^{m}\right)\right) \frac{\partial(u-v)}{\partial t} d x+\int_{\Omega} d^{\alpha}\left(\nabla u^{m}\right. \\
& \left.-\nabla v^{m}\right) \cdot \phi \nabla\left(u^{m}-v^{m}\right) g_{n}^{\prime}\left(\phi\left(u^{m}-v^{m}\right)\right) d x \\
& +\int_{\Omega} d^{\alpha}\left(\nabla u^{m}-\nabla v^{m}\right) \cdot \nabla \phi\left(u^{m}-v^{m}\right) g_{n}^{\prime}\left(u^{m}\right. \\
& \left.-v^{m}\right) d x+\sum_{i=1}^{N} \iint_{Q_{T}}\left[b_{i}\left(u^{m}\right)-b_{i}\left(v^{m}\right)\right] \\
& \cdot\left[\phi_{x_{i}}\left(u^{m}-v^{m}\right)+\phi\left(u^{m}-v^{m}\right)_{x_{i}}\right] \\
& \cdot g_{n}^{\prime}\left(\phi\left(u^{m}-v^{m}\right)\right) d x d t=0 .
\end{aligned}
$$


Discrete Dynamics in Nature and Society

9

Let us analyze every term in (85). By $\left|b_{i}\left(u^{m}\right)-b_{i}\left(v^{m}\right)\right| \leq c \mid u^{m}-$ $v^{m} \mid$ and then according to the definition of the trace, by (83), we have

$$
\begin{aligned}
& \lim _{\lambda \rightarrow 0} \mid \int_{\Omega}\left[b_{i}\left(u^{m}\right)-b_{i}\left(v^{m}\right)\right] g_{n}^{\prime}\left(\phi\left(u^{m}-v^{m}\right)\right)\left(u^{m}-v^{m}\right) \\
& \cdot \phi_{x_{i}}(x) d x\left|\leq \lim _{\lambda \rightarrow 0} \frac{c}{\lambda} \int_{\Omega_{\lambda} \backslash \Omega_{2 \lambda}}\right| b_{i}\left(u^{m}\right)-b_{i}\left(v^{m}\right) \mid \\
& \cdot\left|g_{n}^{\prime}\left(\phi\left(u^{m}-v^{m}\right)\right)\left(u^{m}-v^{m}\right)\right| d x \leq c \int_{\partial \Omega} \mid b_{i}\left(u^{m}\right) \\
& -b_{i}\left(v^{m}\right)\left|d \Sigma \leq c \int_{\Sigma_{m}^{\prime}}\right| u^{m}-v^{m} \mid d \Sigma .
\end{aligned}
$$

Moreover, we can prove that

$$
\begin{gathered}
\lim _{n \rightarrow \infty} \lim _{\lambda \rightarrow 0} \int_{\Omega}\left(b_{i}\left(u^{m}\right)-b_{i}\left(v^{m}\right)\right) g_{n}^{\prime}\left(\phi\left(u^{m}-v^{m}\right)\right) \\
\cdot\left(u^{m}-v^{m}\right)_{x_{i}} \phi(x) d x=0 .
\end{gathered}
$$

In detail, the limitation (87) is established by the following calculations.

$$
\begin{aligned}
& \lim _{\lambda \rightarrow 0} \mid \int_{\Omega}\left(b_{i}\left(u^{m}\right)-b_{i}\left(v^{m}\right)\right) g_{n}^{\prime}\left(\phi\left(u^{m}-v^{m}\right)\right)\left(u^{m}-v^{m}\right)_{x_{i}} \\
& \cdot \phi(x) d x|=| \int_{\Omega}\left(b_{i}\left(u^{m}\right)-b_{i}\left(v^{m}\right)\right) g_{n}^{\prime}\left(u^{m}-v^{m}\right) \\
& \cdot\left(u^{m}-v^{m}\right)_{x_{i}} d x|=| \int_{\left\{x \in \Omega:\left|u^{m}-v^{m}\right|<1 / n\right\}}\left[b_{i}\left(u^{m}\right)-b_{i}\left(v^{m}\right)\right] \\
& \cdot g_{n}^{\prime}\left(u^{m}-v^{m}\right)\left(u^{m}-v^{m}\right)_{x_{i}} d x \mid \\
& \quad \leqslant c \int_{\left\{x \in \Omega:\left|u^{m}-v^{m}\right|<1 / n\right\}}\left|\frac{b_{i}\left(u^{m}\right)-b_{i}\left(v^{m}\right)}{u^{m}-v^{m}}\right|\left|\left(u^{m}-v^{m}\right)_{x_{i}}\right| d x \\
& \quad=c \int_{\left\{x \in \Omega:\left|u^{m}-v^{m}\right|<1 / n\right\}}\left|d^{-(\alpha-1) / 2} \frac{b_{i}\left(u^{m}\right)-b_{i}\left(v^{m}\right)}{u^{m}-v^{m}}\right| \\
& \cdot\left|d^{(\alpha-1) / 2}\left(u^{m}-v^{m}\right)_{x_{i}}\right| d x \\
& \quad \leqslant c\left[\int_{\{x \in \Omega:|u-v|<1 / n\}}\left(\left|d^{-(\alpha-1) / 2} \frac{b_{i}\left(u^{m}\right)-b_{i}\left(v^{m}\right)}{u^{m}-v^{m}}\right|\right)^{2} d x\right]^{1 / 2} \\
& \quad \cdot\left[\int_{\left\{x \in \Omega:\left|u^{m}-v^{m}\right|<1 / n\right\}}\left|d^{\alpha-1} \nabla\left(u^{m}-v^{m}\right)\right|^{2} d x\right]^{1 / 2} \cdot
\end{aligned}
$$

Since $1 \leq \alpha<2$,

$$
\begin{aligned}
& \int_{\left\{x \in \Omega:\left|u^{m}-v^{m}\right|<1 / n\right\}}\left(\left|d^{-(\alpha-1) / 2} \frac{b_{i}\left(u^{m}\right)-b_{i}\left(v^{m}\right)}{u^{m}-v^{m}}\right|\right)^{2} d x \\
& \leqslant c \int_{\Omega} d^{1-\alpha} d x \leqslant c .
\end{aligned}
$$

In (88), let $n \rightarrow \infty$. If $\left\{x \in \Omega:\left|u^{m}-v^{m}\right|=0\right\}$ is a set with 0 measures, then

$$
\begin{aligned}
\lim _{n \rightarrow \infty} & \int_{\left\{x \in \Omega:\left|u^{m}-\nu^{m}\right|<1 / n\right\}} d^{1-\alpha}(x) d x \\
= & \int_{\left\{x \in \Omega:\left|u^{m}-v^{m}\right|=0\right\}} d^{1-\alpha}(x) d x=0 .
\end{aligned}
$$

If the set $\left\{x \in \Omega:\left|u^{m}-v^{m}\right|=0\right\}$ has a positive measure, then, by (22),

$$
\begin{aligned}
& \lim _{n \rightarrow \infty} \int_{\left\{x \in \Omega:\left|u^{m}-v^{m}\right|<1 / n\right\}} d^{\alpha-1}\left|\nabla\left(u^{m}-v^{m}\right)\right|^{2} d x \\
& \quad \leq \int_{\left\{x \in \Omega:\left|u^{m}-v^{m}\right|=0\right\}} d^{\alpha-1}\left(\left|\nabla u^{m}\right|^{2}+\left|\nabla v^{m}\right|^{2}\right) d x=0 .
\end{aligned}
$$

Therefore, in both cases, the right hand side of inequality (88) goes to 0 as $n \rightarrow \infty$.

At the same time, then,

$$
\begin{aligned}
& \lim _{\lambda \rightarrow 0} \int_{\Omega} d^{\alpha}\left(\nabla u^{m}-\nabla v^{m}\right) \cdot \nabla \phi\left(u^{m}-v^{m}\right) g_{n}^{\prime}\left(\phi \left(u^{m}\right.\right. \\
& \left.\left.-v^{m}\right)\right) d x=\lim _{\lambda \rightarrow 0} \int_{\Omega_{\lambda} \backslash \Omega_{2 \lambda}} \frac{c}{\lambda} d^{\alpha}\left|\nabla u^{m}-\nabla v^{m}\right| \\
& \cdot\left|\left(u^{m}-v^{m}\right) g_{n}^{\prime}\left(\phi\left(u^{m}-v^{m}\right)\right)\right| d x \\
& \leq c \lim _{\lambda \rightarrow 0} \int_{\Omega_{\lambda} \backslash \Omega_{2 \lambda}} d^{\alpha-1}\left|\nabla u^{m}-\nabla v^{m}\right| \mid\left(u^{m}-v^{m}\right) \\
& \cdot g_{n}^{\prime}\left(\phi\left(u^{m}-v^{m}\right)\right) \mid d x \\
& \leq c \lim _{\lambda \rightarrow 0}\left(\int_{\Omega_{\lambda} \backslash \Omega_{2 \lambda}}\left[d^{(\alpha-1) / 2}\left|\nabla u^{m}-\nabla v^{m}\right|\right]^{2} d x\right)^{1 / 2} \\
& \cdot\left(\int_{\Omega_{\lambda} \backslash \Omega_{2 \lambda}} \mid d^{(\alpha-1) / 2}\left(u^{m}-v^{m}\right)\right. \\
& \left.\left.\cdot g_{n}^{\prime}\left(\phi\left(u^{m}-v^{m}\right)\right)\right|^{2} d x\right)^{1 / 2} \cdot
\end{aligned}
$$

Since

$$
\begin{aligned}
& \lim _{\lambda \rightarrow 0} \int_{\Omega_{\lambda} \backslash \Omega_{2 \lambda}}\left|d^{(\alpha-1) / 2}\left(u^{m}-v^{m}\right) g_{n}^{\prime}\left(\phi\left(u^{m}-v^{m}\right)\right)\right|^{2} d x \\
& \quad \leq \lim _{\lambda \rightarrow 0} \int_{\Omega}\left|d^{(\alpha-1) / 2}\left(u^{m}-v^{m}\right) g_{n}^{\prime}\left(\phi\left(u^{m}-v^{m}\right)\right)\right|^{2} d x \\
& \quad \leq \int_{\Omega} d^{\alpha-1} d x \leq c,
\end{aligned}
$$

and by (22)

$$
\lim _{\lambda \rightarrow 0} \int_{\Omega_{\lambda} \backslash \Omega_{2 \lambda}}\left[d^{(\alpha-1) / 2}\left|\nabla u^{m}-\nabla v^{m}\right|\right]^{2} d x=0,
$$

then we have

$$
\begin{aligned}
& \lim _{\lambda \rightarrow 0} \int_{\Omega} d^{\alpha}\left(\nabla u^{m}-\nabla v^{m}\right) \\
& \quad \cdot \nabla \phi\left(u^{m}-v^{m}\right) g_{n}^{\prime}\left(\phi\left(u^{m}-v^{m}\right)\right) d x=0 .
\end{aligned}
$$

Clearly,

$$
\begin{gathered}
\lim _{n \rightarrow \infty} \lim _{\lambda \rightarrow 0} \int_{\Omega} g_{n}\left(\phi\left(u^{m}-v^{m}\right)\right) \frac{\partial(u-v)}{\partial t} d x \\
=\frac{d}{d t}\|u-v\|_{1} .
\end{gathered}
$$


Now, after letting $\lambda \rightarrow 0$, let $n \rightarrow \infty$ in (85). Then

$$
\begin{aligned}
& \int_{\Omega}|u(x, t)-v(x, t)| d x \\
& \leqslant \int_{\Omega}\left|u_{0}(x)-v_{0}(x)\right| d x+c \int_{\sigma_{m}^{\prime}}|u-v| d \Sigma \\
& \quad+c \int_{0}^{t} \int_{\Omega}|u(x, t)-v(x, t)| d x d t .
\end{aligned}
$$

By Gronwall Lemma, the stability (23) is true. Theorem 5 is proved.

\section{The BV Solution of Equation}

Recently, Zhan considered the initial-boundary value problem of the following equation in [9]

$$
\frac{\partial u}{\partial t}=\frac{\partial}{\partial x_{i}}\left(a(u, x, t) \frac{\partial u}{\partial x_{i}}\right)+\operatorname{div}(b(u)),
$$

$$
(x, t) \in Q_{T},
$$

with

$$
\begin{aligned}
& u(x, 0)=u_{0}(x), \quad x \in \Omega, \\
& u(x, t)=0, \quad(x, t) \in \Sigma_{p} \times(0, T),
\end{aligned}
$$

where

$$
\begin{aligned}
& \Sigma_{p}=\left\{x \in \Omega: b_{i}^{\prime}(0) n_{i}(x)<0\right\}, \\
& A(u, x, t)=\int_{0}^{u} a(s, x, t) d s, \\
& a(s, x, t) \geq 0, a(0, x, t)=0 .
\end{aligned}
$$

Definition 11. A function $u \in B V\left(Q_{T}\right) \cap L^{\infty}\left(Q_{T}\right)$ is said to be the entropy solution of equation (98) with the initialboundary values (99)-(100), if we have the following.

(1) There exists $g^{i} \in L^{2}\left(Q_{T}\right), i=1,2, \ldots, N$, such that, for any $\varphi(x, t) \in C_{0}^{1}\left(Q_{T}\right)$,

$$
\begin{aligned}
& \iint_{Q_{T}} \varphi(x, t) g^{i}(x, t) d x d t \\
& \quad=\iint_{Q_{T}} \varphi(x, t) \sqrt{a(u, x, t)} \frac{\partial u}{\partial x_{i}} d x d t,
\end{aligned}
$$

where $\sqrt{a(u, x, t)}$ is composite mean value of $\sqrt{a(u, x, t)}$.

(2) For any $\varphi \in C_{0}^{2}\left(Q_{T}\right), \varphi \geq 0$, for any $k \in \mathbb{R}$, and for any small $\eta>0, u$ satisfies

$$
\begin{aligned}
& \iint_{Q_{T}}\left[I_{\eta}(u-k) \varphi_{t}-B_{\eta}^{i}(u, k) \varphi_{x_{i}}+A_{\eta}(u, x, t, k) \Delta \varphi\right. \\
& \left.-\sum_{i=1}^{N} S_{\eta}^{\prime}(u-k)\left|g^{i}\right|^{2} \varphi\right] d x d t \\
& \quad+\iint_{Q_{T}} \int_{k}^{u} a_{x_{i}}(s, x, t) S_{\eta}(s-k) d s \varphi_{x_{i}} d x d t \geq 0 .
\end{aligned}
$$

(3) The boundary condition (100) is true in the sense of trace. The initial value is true in the sense of

$$
\lim _{t \rightarrow 0} \int_{\Omega}\left|u(x, t)-u_{0}(x)\right| d x=0
$$

The existence of the BV solution of equation (98) is by considering the following regularized problem:

$$
\frac{\partial u}{\partial t}=\frac{\partial}{\partial x_{i}}\left(a(u, x, t) \frac{\partial u}{\partial x_{i}}\right)+\varepsilon \Delta u+\sum_{i=1}^{N} \frac{\partial b_{i}(u)}{\partial x_{i}}
$$

in $Q_{T}$,

with the initial-boundary conditions

$$
\begin{aligned}
& u(x, 0)=u_{0}(x), \quad x \in \Omega, \\
& u(x, t)=0, \quad(x, t) \in \partial \Omega \times(0, T) .
\end{aligned}
$$

If there is a constant $\delta>0$ such that

$$
a(\cdot, x, t)-\delta \sum_{s=1}^{N+1}\left(a_{x_{s}}\right)^{2} \geq 0
$$

where $x_{N+1}=t$, then we have the following important estimate.

Theorem 12 (see [9]). Let $u_{\varepsilon}$ be the solution of (105) with (106) and (107). If $a_{x_{i}}=\partial a(u, x, t) / \partial x_{i}$ and $a_{t}$ are all bounded, assumption (108) is true; then

$$
\left|\operatorname{grad} u_{\varepsilon}\right|_{L^{1}(\Omega)} \leq c,
$$

where $|\operatorname{grad} u|^{2}=\sum_{i=1}^{N}\left|\partial u / \partial x_{i}\right|^{2}+|\partial u / \partial t|^{2}$ and $c$ is independent of $\varepsilon$.

By the theorem, we can prove the existence of the entropy solution $u \in B V\left(Q_{T}\right)$ of equation (98) in the sense of Definition 11.

Theorem 13 (see [9]). Suppose that $A(s, x, t)$ is $C^{3}, b_{i}(s)$ is $C^{2}$, $u_{0}(x) \in L^{\infty}(\Omega) \cap C^{1}(\Omega)$, and there is a constant $\delta>0$ such that (108) is true. Then (98) with the initial condition (99) has an entropy solution in the sense of Definition 11. Moreover, let $u, v$ be two solutions of (6) with the initial value $u_{0}(x), v_{0}(x)$ satisfying (7). Then

$$
\begin{aligned}
\int_{\Omega}|u(x, t)-v(x, t)| \leq & \int_{\Omega}\left|u_{0}(x)-v_{0}(x)\right| d x \\
& +\int_{\Sigma_{m}^{\prime}}|u-v| d \Sigma,
\end{aligned}
$$

where $\Sigma_{m}^{\prime}=\partial \Omega \backslash \Sigma_{m}, \Sigma_{m}=\left\{x \in \partial \Omega: b_{i}^{\prime}(0) n_{i}(x)<0\right\}$, and $\left\{n_{i}\right\}$ is the inner normal vector of $\Omega$. In particular, if $b_{i}(s) \equiv 0$, then $\Sigma_{p}=\emptyset$; we have

$$
\int_{\Omega}|u(x, t)-v(x, t)| \leq \int_{\Omega}\left|u_{0}(x)-v_{0}(x)\right| d x .
$$


If $b_{i}(s) \equiv 0$, Theorem 13 implies that the solution of (98) is controlled by the initial condition. In other words, no boundary value condition is needed.

Now, let

$$
a(u, x, t)=m d^{\alpha} u^{m-1}
$$

Then, for any $0 \leq u \leq M$,

$$
\begin{aligned}
a_{t} & =0, \\
a_{x_{i}} & =m \alpha d^{\alpha-1} d_{x_{i}} u^{m-1},
\end{aligned}
$$

by the fact that $|\nabla d|^{2}=1$ a.e. in $\Omega$,

$$
\begin{aligned}
& a(u, x, t)-\delta \sum_{s=1}^{N+1}\left(a_{x_{s}}\right)^{2} \\
& =m d^{\alpha} u^{m-1}-\delta m^{2} \alpha^{2} d^{2 \alpha-2} u^{2(m-1)} \\
& =m d^{\alpha} u^{m-1}\left(1-\delta m \alpha d^{\alpha-2} u^{m-1}\right) .
\end{aligned}
$$

If $\alpha \geq 2, m \geq 1$, there exists $\delta$ such that inequality (108) is true. But, in general, the distance function $d$ only is a continuous function and is differential for almost everywhere in $\Omega$; then

$$
a(s, x, t)=m d^{\alpha} s^{m-1}
$$

does not belong to $C^{3}$, so we can not have Theorems 12 and 13 directly. However, if we check the proof of Theorems 12 and 13, only if we assume that $\Omega$ is a appropriately smooth such that $d_{x_{i}}(x)$ is integral on $\partial \Omega$, then similar to the proof of Theorems 12 and 13, we can prove Theorem 4; we omit the details here.

Remark 14. If $\Omega$ is a $C^{2}$ domain, then $d(x)$ is differential near the boundary $\partial \Omega$, so $d_{x_{i}}(x)$ is a continuous function on $\partial \Omega$ and is integral on $\partial \Omega$.

Remark 15. If $\alpha \geq 2, m \geq 1$, by Theorem $12, u \in \mathrm{BV}\left(Q_{T}\right)$, we can define the trace of $u$ on the boundary $\partial \Omega$.

\section{Conflicts of Interest}

The authors declare that they have no conflicts of interest.

\section{Acknowledgments}

The paper is supported by NSF of China (no. 11371297), supported by NSF of Fujian Province (no. 2015J01592), China.

\section{References}

[1] A. Friedman, Partial differential equations of parabolic type. Prentice Hall, Englewood Cliffs, NJ, USA, 1964.

[2] J. L. Vázquez, Smoothing and Decay Estimates for Nonlinear Diffusion Equations, Equations of Porous Medium Type, Oxford University Press, UK, 2007.

[3] J. Smoller, Shock Waves and Reaction Diffusion Equations, vol. 258, Academic Press, NY, USA, 1983.
[4] A. S. Alexander, A. G. Victor, P. K. Sergei, and P. M. Alexander, Blow-up in quasilinear parabolic equations, Walter de Gruyter, NY, USA, 1995.

[5] Z. Q. Wu, J. N. Zhao, J. X. Yin, and H. Li, Nonlinear Diffusion Equations, World Scientific Publishing, River Edge, NJ, USA, 2001.

[6] E. DiBenedetto, Degenerate Parabolic Equations, Springer, New York, NY, USA, 1993.

[7] J. Yin and C. Wang, "Properties of the boundary flux of a singular diffusion process," Chinese Annals of Mathematics, Series B, vol. 25, no. 2, pp. 175-182, 2004.

[8] J. Yin and C. Wang, "Evolutionary weighted p-Laplacian with boundary degeneracy," Journal of Differential Equations, vol. 237, no. 2, pp. 421-445, 2007.

[9] H. Zhan, "The boundary degeneracy theory of a strongly degenerate parabolic equation," Boundary Value Problems, vol. 2016, no. 1, article no. 15, pp. 1-36, 2016.

[10] H. Zhan, "The solutions of a hyperbolic-parabolic mixed type equation on half-space domain," Journal of Differential Equations, vol. 259, no. 4, pp. 1449-1481, 2015.

[11] H. Zhan, "Homogeneous dirichlet condition of an anisotropic degenerate parabolic equation," Boundary Value Problems, vol. 2015, no. 1, pp. 1-21, 2015.

[12] K. Kobayasi and H. Ohwa, "Uniqueness and existence for anisotropic degenerate parabolic equations with boundary conditions on a bounded rectangle," Journal of Differential Equations, vol. 252, no. 1, pp. 137-167, 2012.

[13] Y. Li and Q. Wang, "Homogeneous dirichlet problems for quasilinear anisotropic degenerate parabolic-hyperbolic equations," Journal of Differential Equations, vol. 252, no. 9, pp. 4719-4741, 2012.

[14] H. S. Zhan and H. J. Yuan, "Porous medium equation with boundary degeneracy," Journal of Jilin University. Science Edition. Jilin Daxue Xuebao. Lixue Ban, vol. 53, no. 5, pp. 829-834, 2015. 


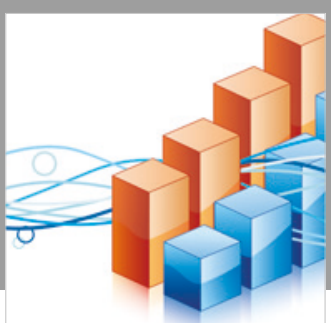

Advances in

Operations Research

\section{-n-m}
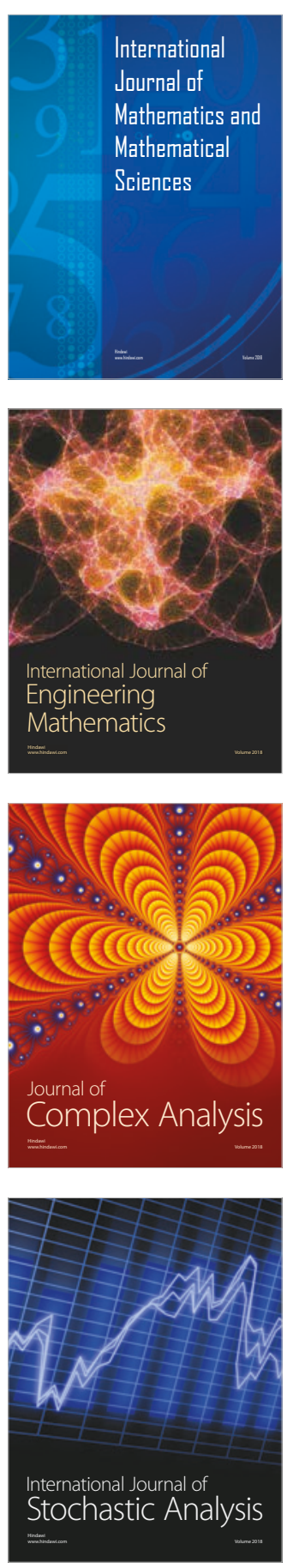
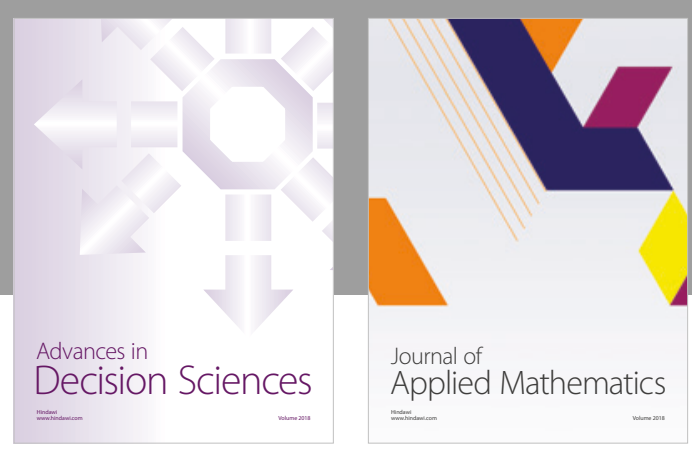

Journal of

Applied Mathematics
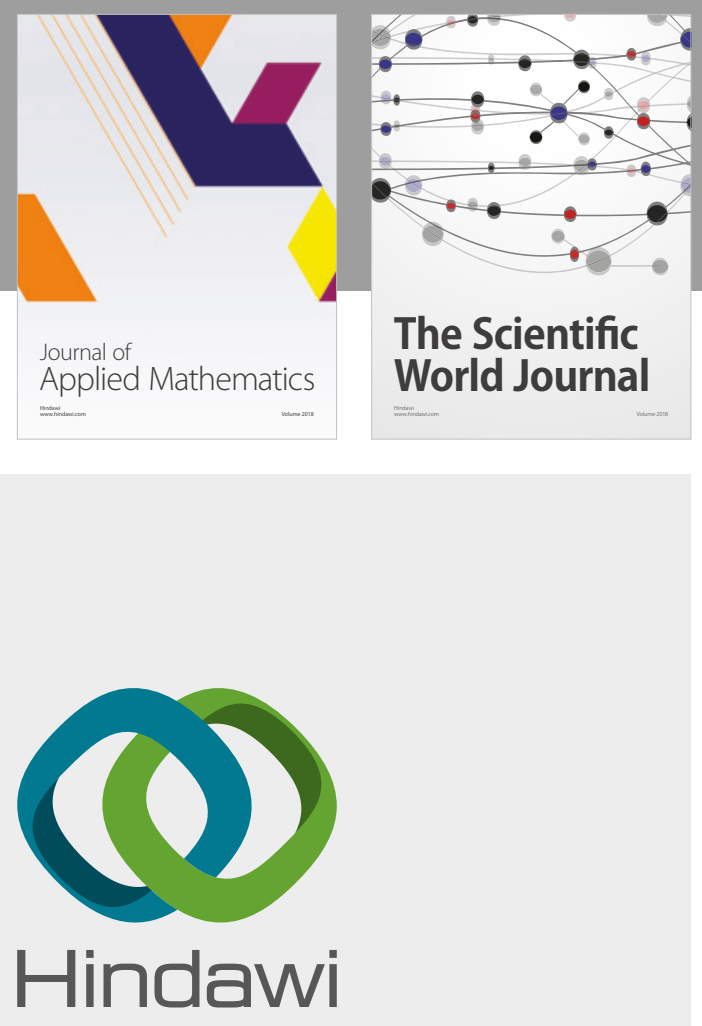

Submit your manuscripts at

www.hindawi.com

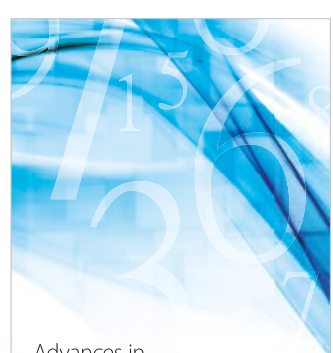

Advances in
Numerical Analysis
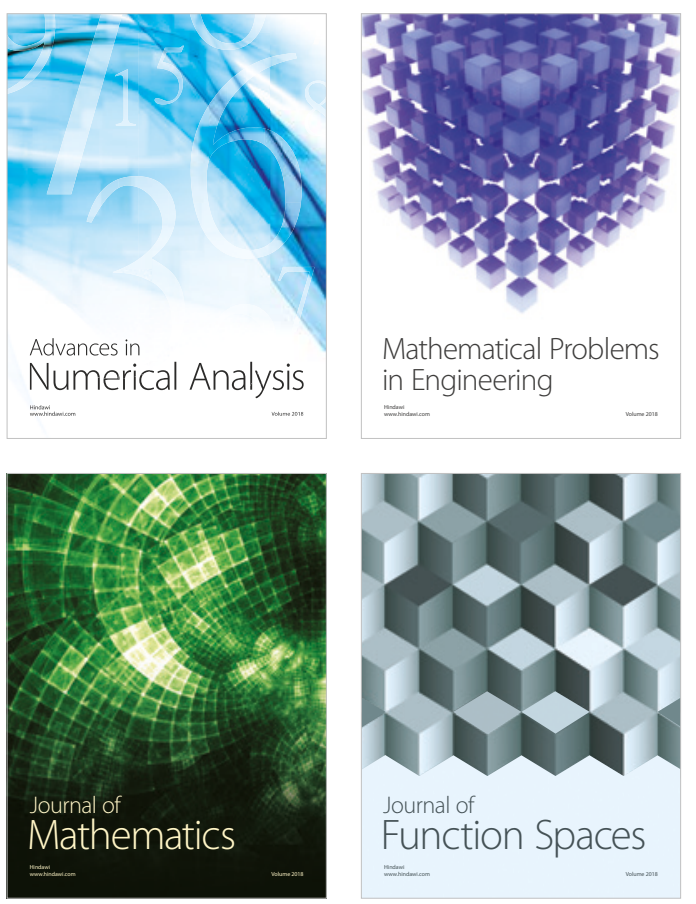

Mathematical Problems in Engineering

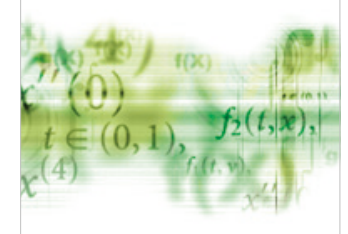

International Journal of

Differential Equations

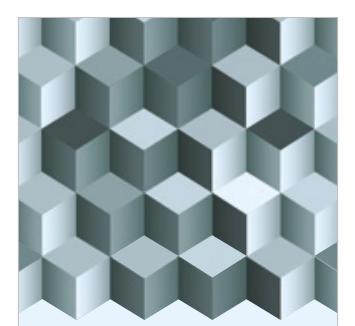

Journal of

Function Spaces

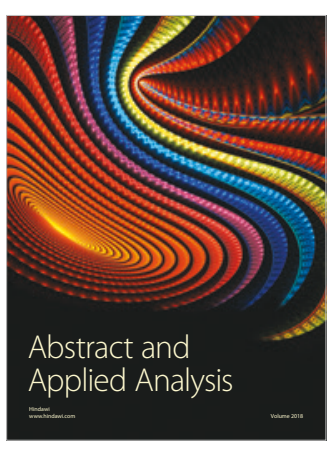

The Scientific

World Journal

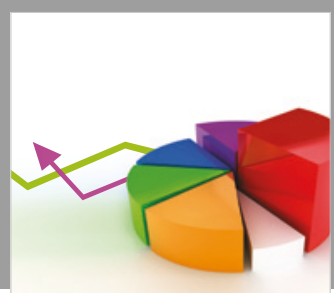

Journal of

Probability and Statistics
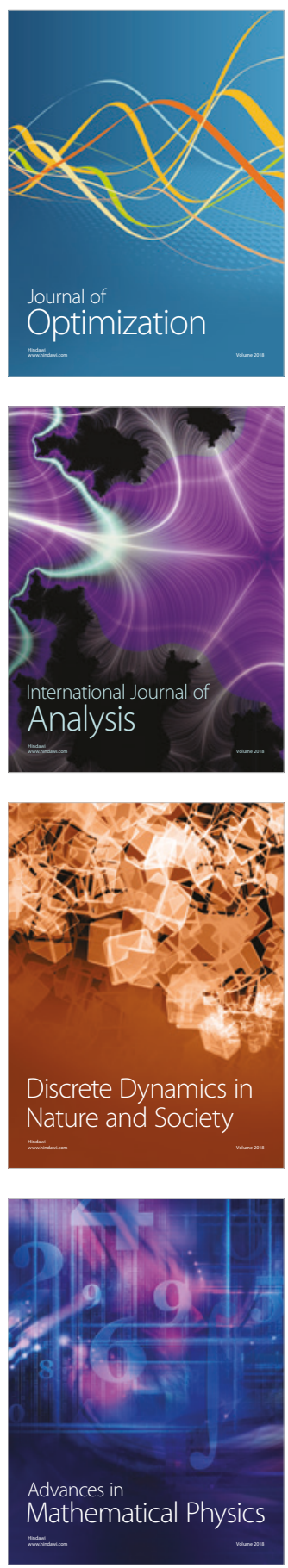